\title{
A Noval Wakeup Schedule Algorithm for Relay Nodes to enhance the lifetime of Sensor Network
}

\author{
Shruti Pandey \\ M. Tech, WCSN, BBDU, Lucknow, UP
}

\begin{abstract}
The appeal of acquiring ambient knowledge to build smart environment results in a discovery of new intelligent devices which have overall abilities of sensing, computation and communication. Through precocious networking protocols, these devices are interconnected to each other and form Wireless Sensor Network (WSN). Routing is gargantuan task in terms of wireless sensor network. A routing protocol for wireless sensor network is different from conspiring it for the traditional networks. Traditional wireless routing protocols do not adapt well to the dynamic wireless environment variations. Hence, these protocols trigger excessive link-level retransmissions, unthriftiness of network resources, and may even lead to system cataclysm. Opportunistic Routing, however, considers the shared wireless medium as an opportunity rather than a limitation. In fact, the key idea behind opportunistic routing is to overcome the drawback of unreliable wireless transmission by taking advantage of the broadcast nature of the wireless medium. The problem arising is of energy optimization in network and if energy is managed, lifetime for the network is enhanced. Thus, we bring the enhanced ENS_OR algorithm, the advanced version of ENS_OR which include wakeup schedule in it. The Enhanced ENS_OR satisfies both metrics manages energy as well as prolong the network lifetime also.
\end{abstract}

Keyword: Wireless sensor network, Opportunistic Routing Protocol, Enhanced ENS_OR, Wakeup schedule.

\section{INTRODUCTION}

Wireless Sensor networks (WSNs) are the network of spatially disseminates sensors which congregate information from the phenomenal world. It is used for audit environmental factors like temperature, pressure and moisture etc. and transmits the information or data to designated nodes [1]. WSN has been proven propitious in number of relevance such as traffic surveillance, military application, weather forecasting, landslide detection, fire detection etc. Each sensor node, as an autonomous device, perceives the physical parameters of the deployment field, processes the data, and then incorporates with data from other nodes in the network to transfer the crucial information to destinations. However, the sensor nodes are usually equipped with restricted resources. In order to successfully fulfill the aims of applications as long as possible, it is very challenging to operate the sensor nodes as well as the overall network efficiently.

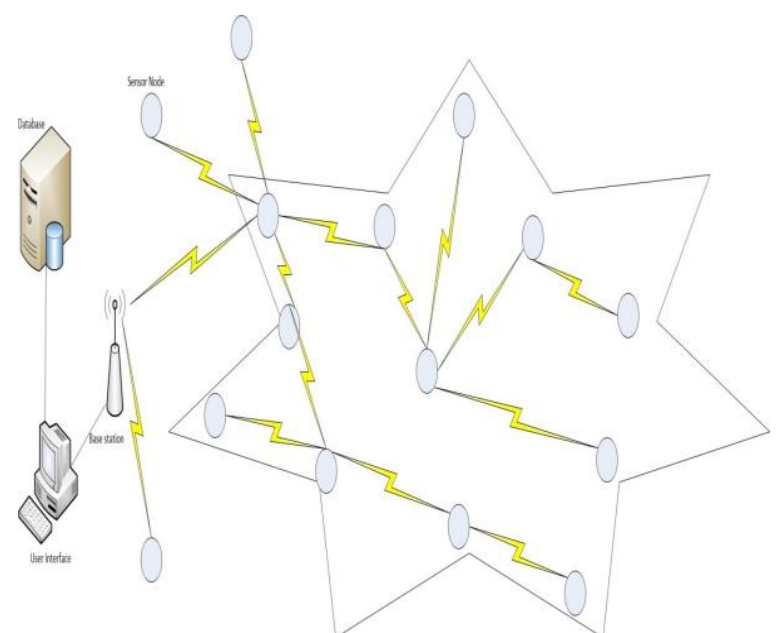

Fig 1: Sensor nodes deployed in the environment

Opportunistic Routing, however, considers the shared wireless medium as an opportunity rather than a limitation. In fact, the key idea behind opportunistic routing is to overcome the drawback of unreliable wireless transmission by taking advantage of the broadcast nature of the wireless medium. That is, instead of pre-selecting a specified relay node at each transmission, opportunistic routing broadcasts a data packet so that it is overheard by multiple neighbors which 
later form the candidate relays set. Then, the actual packet forwarder will be chosen from this set of candidates which have successfully received this data packet. This property is called multi user diversity because it refers to a type of spatial diversity existing across multiple receivers (or users). Opportunistic routing exploits the receptions of the same packet at multiple nodes in order to improve the network performance compared to legacy routing. Indeed, by dynamically selecting the forwarder from a set of multiple receivers, opportunistic routing can significantly reduce the number of packet retransmissions caused by link failures. Thus, robustness is provided by opportunistic routing at a much lower cost (retransmission cost) compared to traditional routing. This main asset, along with other ones, has encouraged the adoption of opportunistic routing in a wide range of recent applications spanning from emergency evacuation and recovery industrial sites interconnection .Internet provision in rural areas to building construction automation and critical infrastructure (water supply, electricity distribution, bridges and railroads, gas/oil pipelines) protection. Clearly, the concept of opportunistic routing can be applied to all kinds of wireless multi-hop networks, such as ad hoc, mesh, and sensor networks, as long as omni-directional antennas are used.

In this paper, we integrate new hybrid routing protocol based on newly proposed wakeup schedule algorithm with WSNs. We consider the particular requirements of WSNs by taking energy efficiency as the main concern and tailoring OR to duty-cycled sensor nodes. The proposed new hybrid routing protocol based on newly proposed wakeup schedule algorithm (Enhanced ENS_OR) selects forwarders by jointly considering multiple types of cross-layer context, such as link quality, progress, residual energy, and energy draining rate. An adaptive duty-cycling scheme has been designed and implemented to tune the sleep intervals of sensor nodes according to traffic load and energy drain rate. Experiment results show that Enhanced ENS_OR improves throughput, and prolongs network lifetime compared to other approaches in static scenarios.

The structure of the paper is as follows. Section II reviews the latest research works. Section III describes the network and energy model. Section IV describes the new hybrid routing protocol based on newly proposed wakeup schedule algorithm (Enhanced ENS_OR) opportunistic routing protocol. Evaluation results and analysis are presented in Section V. The paper concludes with Section VI, which summarizes the contributions of our work, and describes our future works.

\section{RELATED WORK}

In this section, we provide a brief audit of the routing criterion evolution from its inception to its current state. The need for routing standards first arose with emergence of the enterprise networks. The first two main approaches have been developed in this regard, namely Distance Vector Routing (DVR) [21] and Link State Routing (LSR)[22]. In the first approach (DVR), in this radically, each node systematically exchanges its distance vector information with its neighbors in order to arbitrate the next hop node from itself to each destination. The chief asset of this approach is that it only desire local information for the path computation. Despite its ease, in many cases DVR may agonize from slow convergence which leads to the other problems like routing loops. Therefore, this approach fits better for small local area wired networks, where the slow convergence is less likely to happen. The most commonly used DVR-based protocol is the RIP [21].

LSR, on the other hand, needs a global knowledge of network in order to arbitrate the best routing paths. LSR avoids routing loops to prejudice of higher computation complication and higher storage overhead. The most commonly used LSR-based protocol in wired networks is the OSPF [22].

Later, with the proliferation of Internet, the need to optimize these routing approaches has elevated. In this regard, a new technique that advances routing scalability has been developed. This technique is known as Hierarchical Routing [23]. The main attribute of the Hierarchical Routing is that it lies on apportion of the underlying network into altered domains in order to decrease the routing complexity in a large networks like Internet.

Overall, these protocols are based on the traditional routing paradigms which were initially developed for the wired networks. Their main constraints are that they cannot be applied in the applications characterized by intermittent connectivity and anemic infrastructure like the disaster recovery applications. Moreover, the latter proliferation of heterogeneous wireless networks and technologies have created a need to better exploit this distinct potential in order to boost the network capacity and to overcome the deficit of infrastructure in some areas. In the emergence of this new IT context, opportunistic routing drew great heed, within the last decade, benediction to its numerous advantages over traditional routing [7]. Opportunistic Routing Algorithm for Relay Node Selection in Wireless Sensor Networks characterizes the algorithm which cynosure on minimizing the energy consumption of network. The author has proposed Energy Saving via Opportunistic Routing (ENS-OR). The algorithm implements concept of energy efficient node (EEN) which appears to be an implicit relay node obtained by relay function on certain real nodes based on their surplus energy. The forwarder list selection and prioritizing nodes in that ballot is carried out by ENS-OR algorithm measure the optimal hop distance to calculate the next hop node to forward data. The nodes in forwarding list prioritize themselves by their surplus energy and their distance from the EEN.ENS-OR obtain improved network energy usage. Also it enhances the network lifetime by accomplishing higher residual energy of nodes in the network. 
From the analysis of the related work, we claim that ENS_OR protocol considering multiple network contexts is beneficial for low-power WSNs. In this paper, we design a new hybrid opportunistic routing protocol with suitable adaptations to WSN features. To achieve a balance between performance and energy efficiency, our proposal includes an adaptive duty cycling scheme, which controls sensors' sleep intervals according to traffic load and energy drain rate. New hybrid routing protocol based on newly proposed wakeup schedule algorithm in order to enhance lifetime of sensor network. Then to compare the newly proposed wakeup schedule algorithm with standard routing technique.

\section{NETWORK AND ENERGY MODELS:}

In this section, the network model and energy model is described.

\section{A. Network Model}

We consider a multi-hop WSN in a linear queue model as shown in Fig. 2. We assume that our scheme is targeted for relatively dense network, i.e., each relay node has plenty of neighboring nodes .Every wireless sensor node has fixed maximum transmission range Rand minimal transmission range dmin. We set the indices $\{0,1,2, \ldots, \mathrm{h}, \mathrm{n}, \ldots ., \mathrm{M}-$ $1, \mathrm{M}\}$ from left to right, and two specific nodes with index 0 and index Mamong them as the source node and the sink node. Let $\mathrm{N}(\mathrm{h})$ represents as the neighbor set of a node h, i.e., $\mathrm{n} \in \mathrm{N}(\mathrm{h})$.

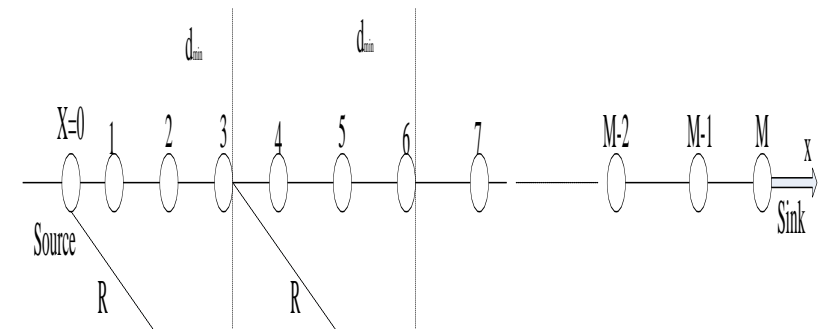

Fig 2: Network topology

\section{B. Energy model}

In this work, we refer to a simplified power model of radio communication as it is used in [20] and [21]. The energy consumption can be expressed as follows:

$$
E_{T}=\left(E_{e l e c}+\varepsilon_{a m p} d^{\tau}\right) \mathrm{B}->\text { (1) }
$$

whereEelec is the basic energy consumption of sensor board to run the transmitter or receiver circuitry, and camp is its energy dissipated in the transmit amplifier. dis the distance between transmitter and receiver, $\tau$ is the channel path-loss exponent of the antenna, which is affected by the radio frequency (RF) environment and satisfies $2 \leq \tau \leq 4$. ETdenotes the energy consumption to transmit a $B$-bit message in a distance $d$.

On the other hand, the energy consumption of receiver $E R$ can be calculated as follows:

$$
E_{R}=E_{e l e c} \mathrm{~B}->(2)
$$

In our model, since the noise and environmental factor are constant, only the transmitter can adjust its transmission power to make $E T$ reach a minimum value.

\section{PROTOCOL DESCRIPTION}

Energy consumption analysis is conducted on the new hybrid routing protocol based on newly proposed wakeup schedule algorithm, where data are delivered to sink node through hop-by-hop connected relay nodes. Our objective is to design new hybrid routing protocol based on newly proposed wakeup schedule algorithm an energy-efficient opportunistic routing strategy for each relay node that ensures minimum power cost and protects the nodes with relatively low residual energy.

For this, first we find the optimize distance on the given formula:

$$
d_{o p}=\left\{\left(2 * E_{\text {elec }}\right) /\left[(\tau-1) \varepsilon_{a m p}\right]\right\}^{1 / \tau}->(3)
$$


For data to be transmitted algorithm selects a forwarder node.This is done as, when the intermediate node has been assumed, the forwarder node has to be within the maximum range $\mathrm{R}$. The distance between the intermediate node and next forwarder node has to be less than optimal transmission distance.If $\mathrm{h}$ is intermediate node and $\mathrm{F}$ is forwarder node than $\mathrm{dmin}<\mathrm{dop}<\mathrm{R}$ The forwarder set from neighboring nodes has been selected.Forwarder set has to be prioritized.

$$
\begin{aligned}
& P(h+i)=\left\{\left(d_{h+i}-d_{h}\right)\left[\frac{1}{\left|d_{h+i}-d_{o p}\right|}+\left(E_{h+i}-\zeta\right)\right]\right. \\
& =\{(h+i) \in F(h),-R \leq i \leq R
\end{aligned}
$$

where $d h+i-d h$ is the distance between node $h$ and neighbor node $h+i, E h+i$ denotes the residual energy of node $h+i$, and $\zeta$ denotes the value of energy threshold.

As the packets have been broadcast, it reaches to its destination. The problem arising in form of managing energy and optimizing the energy and enhancement of network lifetime i.e., nodes stay alive in network for long time.WSN sensors sleep to save energy.

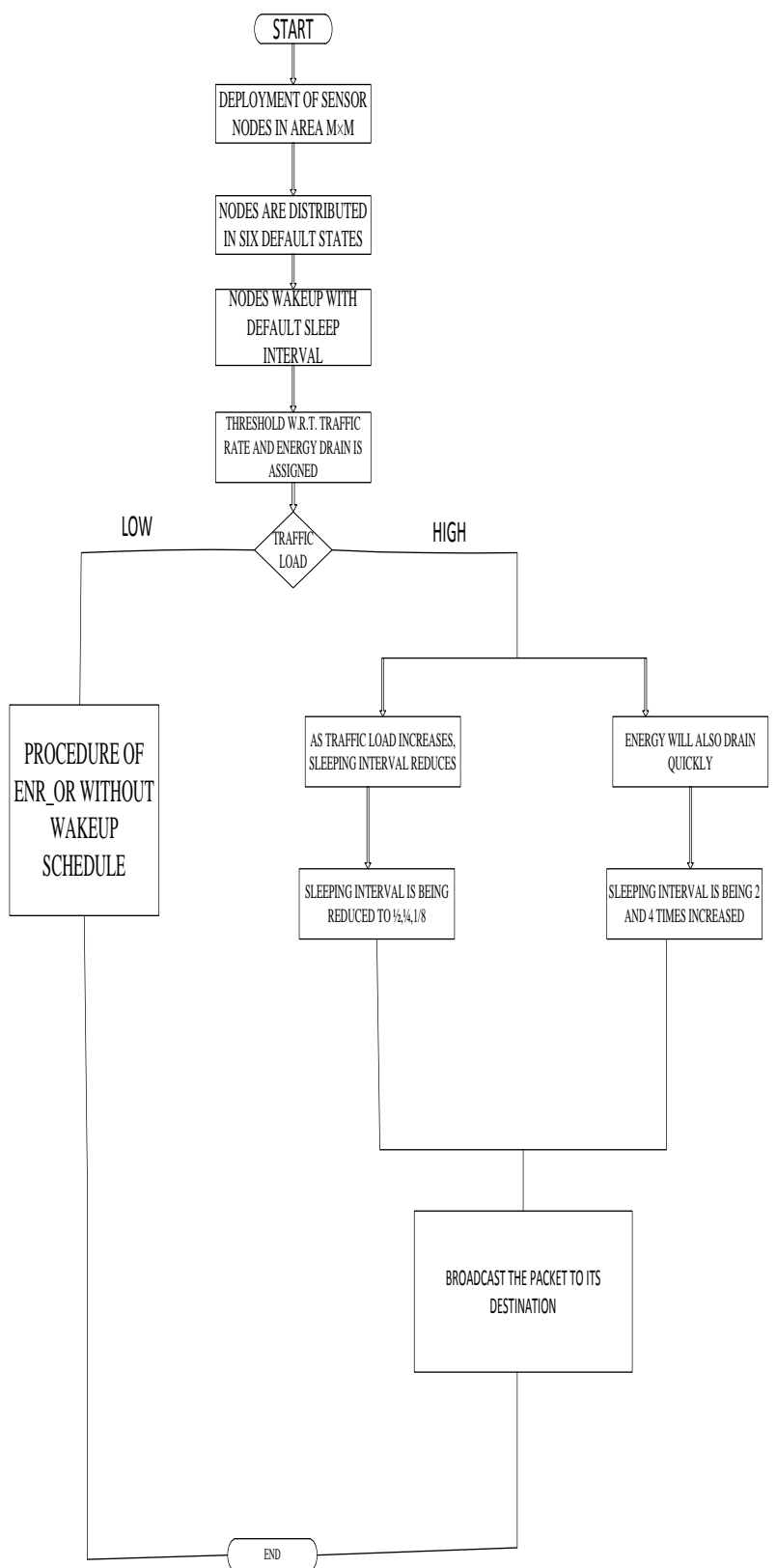

Fig 4: flow chart of Enhanced ENS_OR 
Increasing the sleep interval makes sensor nodes sleep longer and save energy. Sleep interval/wakeup schedule is the on and off state of nodes. When the nodes are awake i.e., their synchronizing the packet and then transmitting it, is the on state. As all the nodes are active and participating in the ENS_OR transmission, energy is draining quickly.To avoid draining of energy and to prolong the network, we introduced the wakeup schedule.

Above Fig illustrate the working of Enhanced ENS_OR, which state that if traffic load is high then nodes distributed in six states wake up from default sleep interval, and their sleep reduces by $1 / 2,1 / 4,1 / 8$. Due to high traffic load the nodes have to be active for long time, so the wakeup schedule increases by 2 and 4 times.

\section{PERFORMANCE EVALUATION IN DIFFERENT METRICS}

Simulation Scenario Experiments:

1) Simulation Environment:We conduct the simulation experiments using MATLAB with 20 nodes uniformly and independently distributed over a line. Each node has the same frequency $B=1 \mathrm{Mbit} / \mathrm{s}$, and firmware character Eelec and $\varepsilon a m p$ in (1) is set as $50 \times 10-9 \mathrm{~J} / \mathrm{bit}$ and $100 \times 10-12 \mathrm{~J} / \mathrm{bit} / \mathrm{m} 2$, respectively. Path-loss exponent of environment $\tau$ is 2 .Hence, the value of optimal transmission distance $d$ op in (3) is approximately equal to $31.6 \mathrm{~m}$. Since Eelec and $\varepsilon$ amp, $\tau$ are fixed, no matter how the distance between two nearest nodes changes, $d$ op still will be $31.6 \mathrm{~m}$, without change. The longest transmission distance of a single hop is $50 \mathrm{~m}$ and the initial energy is $720 \mathrm{~mJ}$. Other simulation parameters are listed in Table I. Network topology one-source-one-sink topology. In this paper, we ignore the interference among the generated signals of each node.

TABLE I Simulation parameter

\begin{tabular}{|l|l|}
\hline Parameter & Parameter's value \\
\hline Deployment Area & $50 \mathrm{~m} \times 2500 \mathrm{~m}$ \\
\hline Deployment distribution & Uniform \\
\hline Distance between two adjacent nodes & $5-25 \mathrm{~m}$ \\
\hline Number of nodes & 20 \\
\hline Source node & 1 \\
\hline Sink node & 1 \\
\hline The longest transmission distance & $50 \mathrm{~m}$ \\
\hline Sending rate & $1 \mathrm{packet} / \mathrm{s}$ \\
\hline Packet size & 1024 bit \\
\hline Eelec & $50 \times 10^{-9}$ \\
\hline Eamp & $100 \times 10^{-12}$ \\
\hline E0 & $720 \times 10^{-2}$ \\
\hline Alpha & 50 \\
\hline Sleep interval & $50,25,12.5,6.25,100,200$ \\
\hline Ec1 & $0.2 \times 10^{-3}$ \\
\hline Ec2 & $0.7 \times 10^{-3}$ \\
\hline T1 & 0.45 \\
\hline T2 & 0.53 \\
\hline T3 & 0.6 \\
\hline
\end{tabular}

2) Performance Metrics:

We define four main measurable metrics to evaluate the effectiveness of ENS_OR algorithm for data forwarding in linear queue networks.

1) Average of residual energy (ARE): Relay nodes left with more average residual energy indicates that all the relay nodes are alive for longer time, which would help to prolong network lifetime.

2) Standard deviation of residual energy (SRE): We use SRE as a metric to quantify the energy balance characteristic of the routing protocol, we have noticed that high standard deviation in the estimations of residual energy implies the unbalanced energy dissipation among sensor nodes, and lowering SRE is important for the routing protocol.

3) First dead node (FDN): We define this metric to evaluate the influence of the network connectivity. As the first energy exhausted node appears, the probability of network partition increases, and the connectivity of the network goes bad.

4) Network lifetime (NL): The network lifetime of a linear queue network is defined as the time when the sink is unable to receive packet sent from the source. The network lifetime is closely related to the energy consumption and network partition. The higher the network lifetime is, the more effectively the balance of energy consumption will be achieved, and the more likely the network partition is going to happen. 
Evaluation of Relay Algorithm:

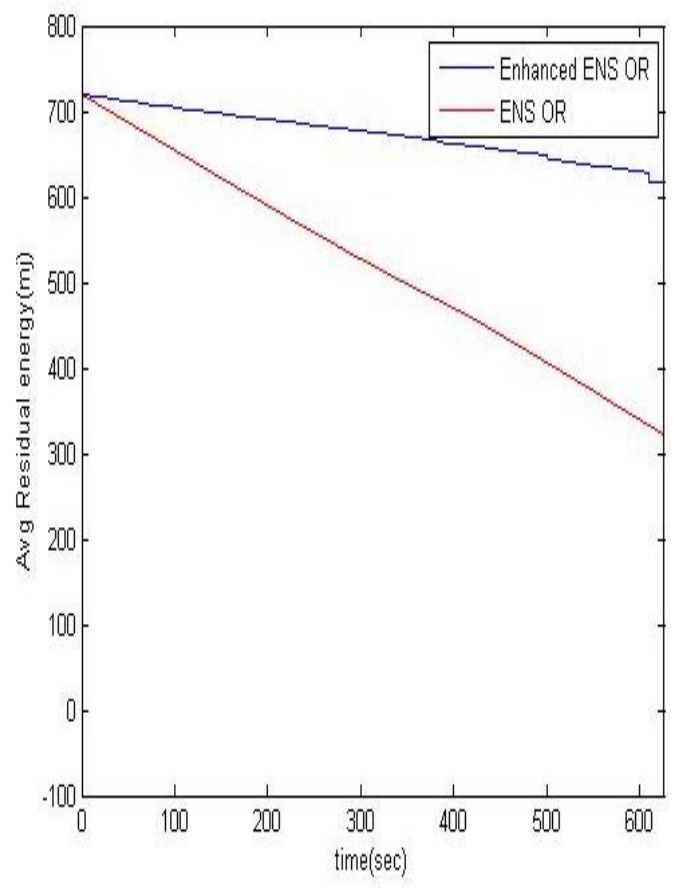

Fig 4: Comparison ARE according to time.

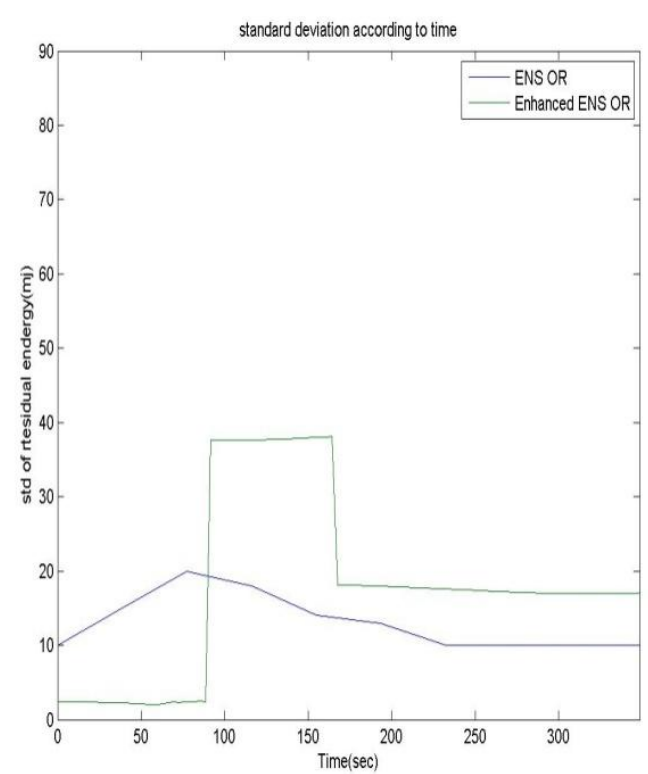

Fig 5: Comparison of SRE according to time

Fig. 4 describes the average residual energy as a function of time, when system is fully operated. As we can see, in general, the total residual energy decreases as the simulation time increases. This can be explained by (1) and (2), where packet size grows incrementally over time can communicate with more energy over a given distance. Enhanced ENS_OR can achieve higher average residual energy compared with ENS_OR, because of its energy optimal strategy and opportunistic routing scheme. Enhanced ENS_OR always keeps the energy consumption at the lowest level. Due to the lower energy consumption, a longer lifetime can be achieved as well by Enhanced ENS_OR method.

From Fig. 5, we notice that Enhanced ENS_OR has a lower standard deviation of residual energy compared with ENS_OR. 


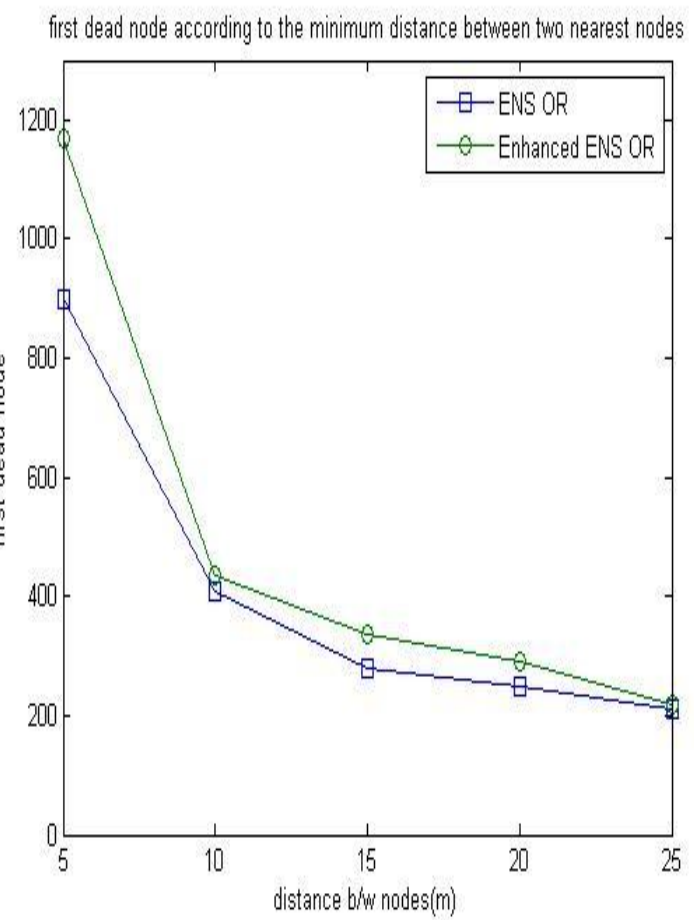

Fig 6: Comparison of FDN according to the minimum distance between two nearest nodes.

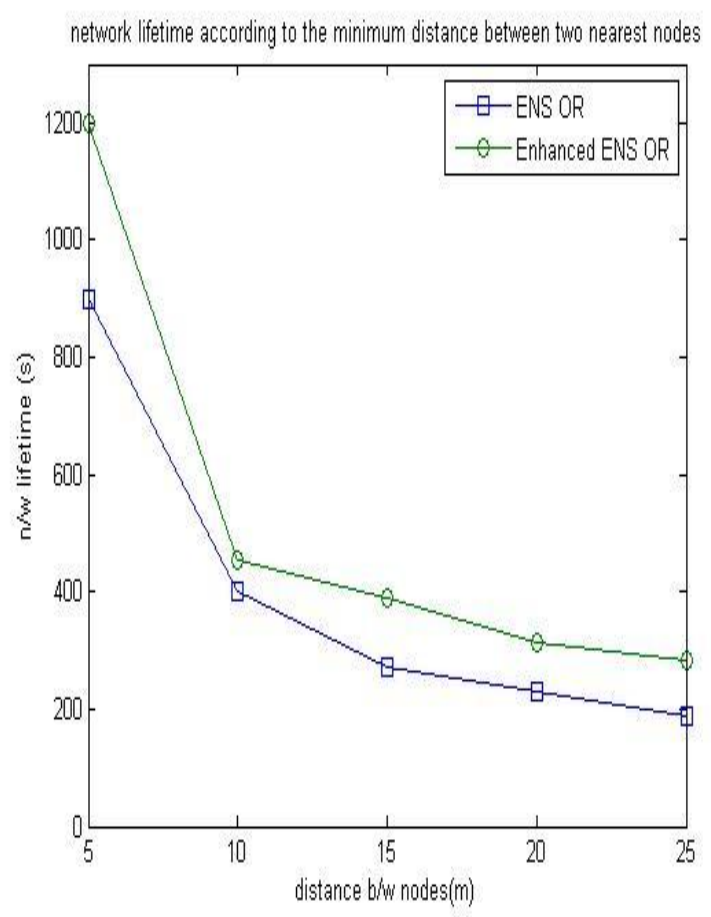

Fig 7: Comparison of NL according to the minimum distance between two nearest nodes.

There is a very strong correlation between FDN and NL. The longer the network lifetime is, and the more slowly the first dead node is going to appear. As shown in Figs. 6 and 7, the result shows that the time that the first dead node appears in Enhanced ENS_OR is much later than that ENS_OR, and the life time of Enhanced ENS_OR is much longer.

Since the optimal energy strategy will especially protect the low energy nodes, ENS_OR performs the best. Thus, ENS_OR guarantees both the extensive lifetime and the largest conservation of energy. 
Vol. 6, Issue 5, May 2017

\section{CONCLUSION AND FUTURE WORK}

WSN has been widely used for monitoring and control applications in our daily life due to its promising features, such as low cost, low power, easy implementation, and easy maintenance. However, most of sensor nodes are equipped with the limited non-rechargeable battery power. Energy savings optimization, therefore, becomes one of major concerns in the WSN routing protocol design.

In this paper, we focus on minimizing energy consumption and maximizing network lifetime of linear queue network where sensors' locations are predetermined and unchangeable.

For this matter, we borrow the knowledge from opportunistic routing theory to optimize the network energy efficiency by considering the differences among sensor nodes in terms of both their distance to sink and residual energy of each other.

We implement opportunistic routing theory to virtually realizethe relay node when actual relay nodes are predeterminedwhich cannot be moved to the place according to the optimal transmission distance. This will prolong the lifetime of the network. Hence, our objective is to design an energy-efficient opportunistic routing strategy that ensures minimum power is cost and protects the nodes with relatively low residual energy. Numerous simulation results show that the proposed solution Enhanced ENS_OR makes significant improvements in energy saving and network partition as compared with other existing routing algorithms.

In future, the proposed algorithm can be worked in heterogeneous environment .

\section{REFERENCES}

[1]. A Survey on Opportunistic Routing Protocols for Wireless Sensor Networks Payal Jadhav*, Prof. RachnaSatao.

[2]. XiaohuaXu, Student Member, IEEE, Xiang-Yang Li, Senior Member, IEEE, and Huadong Ma, Senior Member.” Energy-Efficient Opportunistic Routing in Wireless Sensor Networks"IEEE Transaction on Parallel and Distributed Systems, VOL. 22, NO. 11, Nov 2011.

[3]. Haitao Liu and Baoxian Zhang, Chinese Academy of Sciences Hussein T. Mouftah, University of Ottawa XiaojunShen, University of Missouri - Kansas CityJian Ma, Nokia Research Center "Opportunistic Routing for Wireless Ad Hoc and Sensor Networks: Present and Future Directions "IEEE Transaction on communication magazine, VOL. 22, NO. 11, Dec 2009.

[4]. H. Liu, B. Zhang, H. Mouftah, X. Shen, and J. Ma, "Opportunistic routing for wireless ad hoc and sensor networks: Present and future directions," IEEE Commun. Mag., vol. 47, no. 12, pp. 103-109,Dec. 2009

[5]. Che-Jung Hsu a, Huey-Ing Liu b*, Winston K.G. Seah," Opportunistic Routing - A Review and the Challenges Ahead", IEEE Transaction on Parallel and Distributed Systems, VOL. 22, NO. 11, Nov 2014.

[6]. M. Zorzi and R.R. Rao, "Geographic Random Forwarding (GeRaF) for Ad Hoc and Sensor Networks:Energy and Latency Performance,"IEEE Trans. Mobile Computing, vol. 2, no. 4, Oct.-Dec. 2003.

[7]. S. Biswas and R. Morris, "ExOR: Opportunistic multi-hop routing for wireless networks," in Assoc. Comput. Mach. SIGCOMM Comput. Commun. Rev., 2005, vol. 35, no. 4, pp. 133-144.

[8]. D. De Couto, D. Aguayo, J. Bicket, and R. Morris, "A high-throughput path metric for multi-hop wireless routing In Proc. ACM/IEEE MobiCom, September 2003.

[9]. YanhuaLi , Wei Chen, and Zhi-Li Zhang," Optimal Forwarder List Selection in Opportunistic Routing”Mobile Ad hoc and Sensor Systems. IEEE 6th international conference.

[10]. X. Zhang and B. Li. Dice: “a game theoretic framework for wireless multipath network coding”. In Proceedings of ACM MobiHoc, 2008.

[11]. Goo Yeon Lee and Zygmunt J. Haas,"Simple, Practical, and Effective Opportunistic Routing for Short-Haul Multi-Hop Wireless Networks", IEEE Transaction on Wireless Communication, VOL. 10, NO. 11, Nov 2011.

[12]. Shih-Chun Lin and Kwang-Cheng Chen"Spectrum Aware Opportunistic Routing in Cognitive Radio Networks". IEEE Globecom 2010 proceedings.

[13]. S. Diggavi, N. Al-Dhahir, A. Stamoulis, and A. Calderbank, "Great expectations: The value of spatial diversity in wireless networks," Proc.IEEE, vol. 92, no. 2, pp. 219-270, Feb. 2004.

[14]. X. Qin and R. Berry, "Exploiting multiuser diversity for medium access control in wireless networks," in Proc. IEEE Conf. INFOCOM, 2003, pp. 1084-1094.

[15]. Gorbil and E. Gelenbe, "Resilient emergency evacuation using opportunistic communications," in Proc. 27th ISCIS, Oct. 2012,pp. $249-257$.

[16]. Martin-Campillo, J. Crowcroft, E. Yoneki, and R. Marti, "Evaluating opportunistic networks in disaster scenarios," J. Netw. Comput. Appl., vol. 36, no. 2, pp. 870-880, Mar. 2013.

[17]. S. Yoon, S. Jang, Y. Kim, and S. Bahk, "Opportunistic routing for smart grid with power line communication access networks," IEEE Trans.Smart Grid, vol. 5, no. 1, pp. 1949-3053, Jan. 2014.

[18]. Pentland, R. Fletcher, and A. Hasson, "DakNet: Rethinking connectivity in developing nations," IEEE Comput. Mag., vol. 37, no. 1, pp. 78- 83, Jan. 2004.

[19]. K. Zeng, W. Lou, H. Zhai, Capacity of opportunistic routing in multi-rate and multi-hop wireless networks, IEEE Transactions on Wireless Communications, 7 (2008) 5118-5128.

[20]. C.-P. Luk, W.-C. Lau, O.-C. Yue, An Analysis of Opportunistic Routing in Wireless Mesh Network, in: Proceedings of the IEEE International Conference on Communications (ICC), Beijing, China, 2008.

[21]. C. Hedrick, "Routing information protocol," Internet Requests for Comments, RFC Editor, RFC 1058, Jun. 1988

[22]. J. Moy, “OSPF version 2,” Internet Requests for Comments, RFC Editor, RFC 2178, Jul. 1997

[23]. A Survey on Opportunistic Routing in Wireless Communication Networks Nessrine Chakchouk, Member, IEEEIEEE COMMUNICATION SURVEYS \& TUTORIALS, VOL. 17, NO. 4, FOURTH QUARTER 2015.

[24]. T. Clausen and P. Jaquet, "Optimized Link State Routing Protocol (OLSR)," Internet Requests for Comments, RFC Editor, RFC 3626, Oct. 2003.

[25]. C. Perkins, E. Belding-Royer, and S. Das, “Ad Hoc On-Demand Distance Vector (AODV) routing," Internet Requests for Comments, RFC Editor, RFC 3561, Jul. 2003

[26]. D. Johnson, Y. Hu, and D. Maltz, “The Dynamic Source Routing Protocol (DSR) for mobile ad hoc networks for IPv4," Internet Requests for Comments, RFC Editor, RFC 4728, Feb. 2007. 